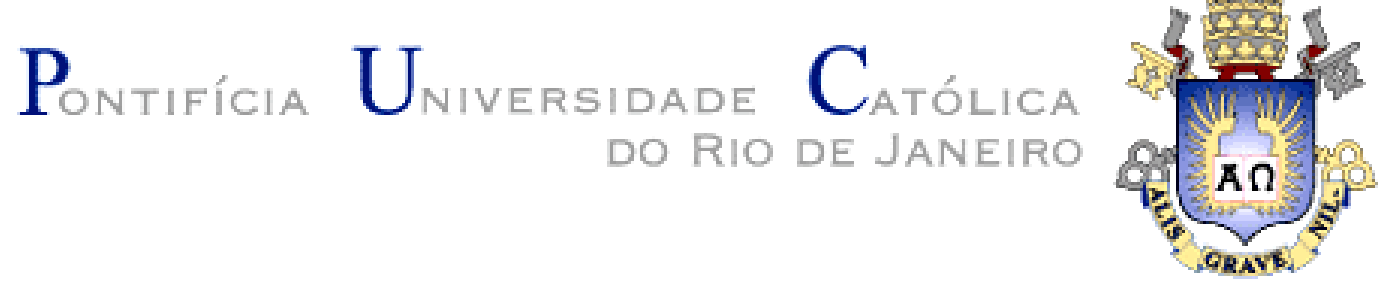

Bianca Fernandes Lima

Simulação de Reservatórios Naturalmente Fraturados

Dissertação de Mestrado

Dissertação apresentada como requisito parcial para obtenção do grau de Mestre pelo Programa de PósGraduação em Engenharia Civil do Departamento de Engenharia Civil da PUC-Rio.

Orientador: Prof. Sérgio Augusto Barreto da Fontoura Co-orientador: Dr. Nelson Inoue 


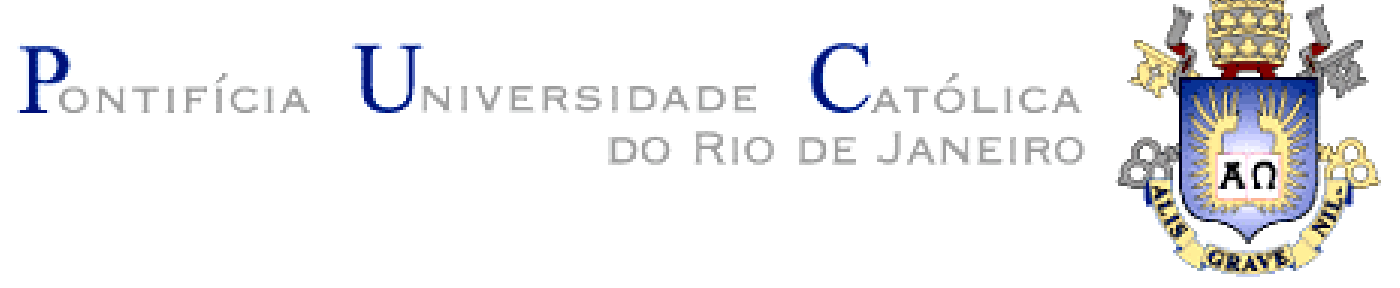

Bianca Fernandes Lima

\section{Simulação de Reservatórios Naturalmente Fraturados}

Dissertação apresentada como requisito parcial para obtenção do grau de Mestre pelo Programa de PósGraduação em Engenharia Civil do Departamento de Engenharia Civil do Centro Técnico Científico da PUCRio. Aprovada pela Comissão Examinadora abaixo assinada.

Prof. Sérgio Augusto Barreto da Fontoura

Orientador

Departamento de Engenharia Civil / PUC-Rio

Dr. Nelson Inoue

Co-orientador

GTEP / PUC-Rio

Profa. Deane Mesquita Roehl

Departamento de Engenharia Civil / PUC-Rio

Dr. Elton Jorge Bragança Ribeiro CENPES / PETROBRAS

Prof. José Eugenio Leal Coordenador Setorial do Centro

Técnico Científico / PUC-Rio

Rio de Janeiro, 14 de março de 2013 
Todos os direitos reservados. É proibida a reprodução total ou parcial do trabalho sem autorização da universidade, do autor e dos orientadores.

\section{Bianca Fernandes Lima}

Graduou-se em Engenharia de Petróleo pela UGF (Universidade Gama Filho - Rio de Janeiro - Brasil) em 2010, tendo sido bolsista integral do Prouni (Programa Universidade para Todos). Entre 2009 e 2010 atuou como estagiária na ANP (Agência Nacional do Petróleo, Derivados e Gás Natural).

Ficha Catalográfica

Lima, Bianca Fernandes

Simulação de reservatórios naturalmente fraturados / Bianca Fernandes Lima ; orientador: Sérgio Augusto Barreto da Fontoura ; co-orientador: Nelson Inoue. 2013.

120 f. il. (color.) ; $30 \mathrm{~cm}$

Dissertação (mestrado)-Pontifícia Universidade Católica do Rio de Janeiro, Departamento de Engenharia Civil, 2013.

Inclui bibliografia

1. Engenharia civil - Teses. 2. Reservatórios fraturados. 3. Dupla-porosidade. 4. Dupla-permeabilidade. 5. Corredores de fraturas. I. Fontoura, Sérgio Augusto Barreto da. II. Inoue, Nelson. III. Pontifícia Universidade Católica do Rio de Janeiro. Departamento de Engenharia Civil. IV. Título. 
Dedico esta dissertação aos meus pais: Gonçala Fernandes Lima e José Alves de Lima. 


\section{Agradecimentos}

Primeiramente, agradeço a Deus pelo seu imenso amor, por me abençoar e por guiar todos os meus passos. A minha vida está nas mãos do Senhor. Agradeço a Deus por realizar maravilhas em minha vida e por colocar pessoas igualmente maravilhosas em meu caminho, verdadeiros anjos. Obrigada Senhor.

Agradeço ao meu orientador, Prof. Sérgio Fontoura, pela confiança depositada desde o dia em que fomos apresentados. Obrigada Prof. Sergio por essa incrível oportunidade de crescimento profissional e pessoal, e pela sua excelente orientação deste trabalho. Foi um grande aprendizado trabalhar com o senhor.

Agradeço ao meu co-orientador, Dr. Nelson Inoue, pelo apoio e incentivo, pelas palavras certas nos momentos oportunos. A sua paciência e a sua dedicação à pesquisa são exemplos a serem seguidos. Foi um prazer trabalhar com você.

Agradeço aos amigos da equipe do GTEP, Grupo de Tecnologia e Engenharia de Petróleo, localizada no Colégio São Marcelo: Guilherme Righetto, Carlos Emmanuel Lautenschläger, Sergio Orozco, Juan David Velilla, Darwin Mateus Tarazona, Pamela Alessandra Rodríguez, Carla Carrapatoso e Ingrid Milena Reyes, por me receberem muitíssimo bem, pelo companheirismo, incentivo e colaboração no desenvolvimento deste trabalho. $\mathrm{E}$ aos demais amigos do GTEP que direta ou indiretamente tiveram participação neste trabalho.

Agradeço, especialmente, à geóloga Paola Rosas, pelo companheirismo, pela amizade construída dia a dia ao longo do ano de 2012, e por todos os momentos de descontração, de alegria, de dúvidas e de compartilhamento de ideias.

Agradeço aos amigos do Departamento de Engenharia Civil, alunos e funcionários, especialmente às amigas Manuella Galindo, Ingrid Magalhães, Mariana Benessiut, Camyla Oliveira e Roberta Soares. Agradeço aos amigos da sala de estudos 611 e a todos que participaram da minha trajetória em busca desta realização. Muitos desses amigos não terão seus nomes relatados nessas linhas, porém eles foram fundamentais nessa conquista e terão um espaço especial reservado em minha memória. “Um homem não constrói sua trajetória sozinho." 
Quero expressar meu agradecimento todo especial à minha grande amiga, Nathalia Christina Passos, pela amizade que construímos desde o nosso primeiro dia de PUC. Obrigada Nathalia, minha dupla, por todos os sentimentos compartilhados, pelo apoio, incentivo, por todos os momentos de estudos e de alegrias.

Sou grata aos professores do Departamento de Engenharia Civil/Geotecnia por todo o conhecimento ministrado, pelos desafios propostos e por todo o enriquecimento profissional e pessoal que me proporcionaram.

Eu não poderia deixar de agradecer a duas outras pessoas que tiveram participação fundamental na conquista desse objetivo. Obrigada amigo, e eterno professor, Dr. Elton Jorge Bragança Ribeiro, por acreditar e confiar em meu trabalho e dedicação e por me conduzir a esta grande oportunidade. Serei eternamente grata ao senhor. E agradeço também ao meu grande amigo, Dr. Arturo Rodrigo Ferreira Pardo, pelo grande apoio em momentos cruciais da construção deste trabalho. Obrigada Arturo por mais uma vez me mostrar sua imensa generosidade.

Agradeço ao CNPQ pela bolsa de estudos concedida a fim de me permitir dedicação total aos estudos.

Para concluir, agradeço a minha família por me apoiar, me incentivar, me levantar nos momentos de dificuldades, por todo de carinho, amor e compreensão. Tudo que sou devo a vocês. E agradeço ao meu namorado Douglas pelo amor e companheirismo de sempre.

E obrigada minha mãezinha do céu, Aparecida, que sempre me rege, me guarda, me protege e me ilumina. 


\section{Resumo}

Lima, Bianca Fernandes; Fontoura, Sérgio Augusto Barreto da; Inoue, Nelson. Simulação de Reservatórios Naturalmente Fraturados. Rio de Janeiro, 2013. 120 p. Dissertação de Mestrado - Departamento de Engenharia Civil, Pontifícia Universidade Católica do Rio de Janeiro.

A presença de fraturas naturais em reservatórios de petróleo pode gerar grandes impactos no deslocamento de fluidos em seu interior. Os maiores problemas estão na irregularidade da área varrida pelo fluido injetado, por exemplo, a água, e a antecipação dos breakthroughs, ou seja, a chegada precoce da água aos poços produtores. De modo a analisar o efeito promovido pela presença de fraturas no meio, foram simulados os dois modelos de simulação de fluxo em reservatórios fraturados: o modelo de dupla-porosidade (DP) e o modelo de dupla-porosidade e dupla-permeabilidade (DPDK), bem como outros dois modelos derivados do modelo DP, o modelo de Subdomínios (SD) e o modelo MINC (Multiple Interacting Continua). Também foram realizadas análises paramétricas para compreender o impacto de propriedades, como as permeabilidades tanto da matriz quanto da fratura e o espaçamento das fraturas, no comportamento do fluxo em reservatórios fraturados. A presença de corredores de fraturas (fracture swarms) foi, igualmente, avaliada, assim como seu efeito associado à presença de camadas de altíssima permeabilidade (super-K). Por fim, foi simulado um modelo mais complexo, denominado GTEP, o qual foi construído a partir de uma seção aplicada à simulação de um reservatório carbonático gigante do Oriente Médio, com o intuito de exemplificar a irregularidade da área varrida em reservatórios naturalmente fraturados.

\section{Palavras-chave}

Reservatórios fraturados; dupla-porosidade; dupla-permeabilidade; corredores de fraturas. 


\section{Abstract}

Lima, Bianca Fernandes; Fontoura, Sérgio Augusto Barreto da (Advisor); Inoue, Nelson (Co-Advisor). Naturally Fractured Reservoirs Simulation. Rio de Janeiro, 2013. 120 p. MSc. Dissertation - Departamento de Engenharia Civil, Pontifícia Universidade Católica do Rio de Janeiro.

The presence of natural fractures in oil reservoirs can generate major impacts on the fluid displacement inside them. The greatest problems are irregularity in the area swept by the injected fluid, e.g. water, and breakthroughs anticipation, or early arrival of water to the producing wells. In order to analyze the effect of the presence of fractures in the medium, two flow models of fractured reservoirs were constructed: the dual-porosity model (DP) and the dualporosity and dual permeability model (DPDK), as well as two other models derived from the DP model, Subdomains model (SD) and MINC model (Multiple Interacting Continua). Parametric analyzes were also conducted to comprehend the impact of properties, such as the matrix permeability, the fracture permeability and the fracture spacing, on the flow behavior in fractured reservoirs. The presence of fracture corridors (fracture swarms) was also evaluated in three models, as well as its effect associated with the presence of layers of high permeability, denoted super-K. Finally, a more complex model, called GTEP Field, was simulated, which was constructed from a section applied to the simulation of a giant carbonate reservoir in the Middle East, in order to illustrate the irregularity of the swept area in natural fractured reservoirs.

\section{Keywords}

Fractured reservoirs; dual-porosity; dual-permeability; fracture corridors. 


\section{Sumário}

1 Introdução 19

1.1. Relevância e motivação do estudo 19

1.2. Objetivo e metodologia 20

1.3. Organização da dissertação 21

2 Revisão bibliográfica $\quad 22$

2.1. Reservatórios naturalmente fraturados 22

2.1.1. Definição de um reservatório naturalmente fraturado 22

2.1.2. Indícios de um reservatório naturalmente fraturado 24

2.1.3. Classificação de reservatórios naturalmente fraturados 26

2.1.4. Processos de recuperação em reservatórios naturalmente fraturados 29

2.1.5. Caracterização e modelagem 35

2.1.6. Importância relativa entre a permeabilidade da matriz e a permeabilidade do sistema de fraturas $\quad 51$

2.1.7. Importância das tensões na variação da permeabilidade das fraturas 51

2.2. Métodos de simulação de reservatórios naturalmente fraturados 54

2.2.1. Modelo de dupla-porosidade - DP 56

2.2.2. Modelo de dupla-porosidade e dupla-permeabilidade - DPDK 61

2.2.3. Termo de transferência de fluxo entre a matriz e as fraturas 62

2.2.4. Variações do modelo de dupla-porosidade - Subdomínio e MINC 68

2.3. Selecionando um modelo de simulação de fluxo 71

3 Metodologia $\quad 75$

3.1. Etapa 1 - Comparação entre os modelos DP e DPDK 77

3.2. Etapa 2 - Análise Paramétrica 82

3.3. Etapa 3 - Corredores de fraturas e camadas de super-permeabilidade (super-K) 85

3.4. Etapa 4 - Modelos alternativos de dupla-porosidade 90

3.5. Etapa 5 - Modelo Campo GTEP 90 
4 Resultados e discussão $\quad 94$

4.1. Resultados Etapa 1 - Comparação entre os modelos DP e DPDK 94

4.2. Resultados Etapa 2 - Análise Paramétrica 97

4.3. Resultados Etapa 3 - Corredores de fraturas e camadas de super$\begin{array}{ll}\text { permeabilidade (super-K) } & 101\end{array}$

4.4. Resultados Etapa 4 - Modelos alternativos de dupla-porosidade 108

4.5. Resultados Etapa 5 - Modelo Campo GTEP 110

5 Conclusões 113

5.1. Trabalhos Futuros 116

$\begin{array}{ll}\text { Referências } & 117\end{array}$ 


\section{Lista de figuras}

Figura 2. 1 - Esquema da porcentagem de porosidade do reservatório versus a porcentagem de permeabilidade do reservatório (porcentagem devido à matriz contra a porcentagem devido à fratura) segundo a classificação de reservatórios naturalmente fraturados de Nelson (2001).

Figura 2. 2 - Recuperação de óleo para reservatórios molháveis à água $\left(I_{a w}=1,0\right)$ e não molháveis à água $\left(I_{a w}=0,09\right)$ Firoozabadi $(2000)$. .33

Figura 2. 3 - Aumento da sucção (pressão capilar) para reservatório não molháveis à água $\left(I_{a w}=0,09\right)$ Firoozabadi $(2000)$. 33

Figura 2. 4 - Componentes de um reservatório naturalmente fraturados (Phelps e Strauss, 2002).

Figura 2. 5 - Metodologia integrada para o desenvolvimento de reservatórios naturalmente fraturados (adaptado de Bourbiaux et al., 2005) 39

Figura 2. 6 - Construção de um modelo de fraturas em multi-escalas (modelo DFN) (Bourbiaux et al., 2005). 40

Figura 2. 7 - Simulação de um teste de build-up no modelo geológico discreto de um setor do reservatório fraturado (Bourbiaux et al., 2005) 43

Figura 2. 8 - Extensão do teste de pressão e sua derivada: teste de curta (early), média (middle) e longa (late) duração (Rawnsley e Wei, 2001).

Figura 2. 9 - Diferença de comportamento entre um meio não fraturado (Well 1) e um meio fraturado (Well 2) (Limsukhon et al., 2009).

Figura 2. 10 - Exemplo de um modelo discreto de fraturas (Weber et al., 2001).

Figura 2. 11 - Resultado do processo de validação e calibração hidráulica aplicado ao modelo discreto de fraturas apresentado na Figura 2. 10: mapa de permeabilidade da fratura (acima) e mapa de espaçamento das fraturas (abaixo) (Weber et al., 2001). 45

Figura 2. 12 - Detalhe do modelo de fraturas: região próxima ao poço e região do upscale de propriedades utilizada para a simulação do teste de fluxo (Le Maux et al., 2005). 46

Figura 2. 13 - Transferência de parâmetros equivalentes do modelo discreto de fraturas para o modelo de simulação de fluxo de um simulador convencional (Adaptado de Al-Muftah et al., 2009). 
Figura 2. 14 - Upscaling do sistema de fraturas discretas para um modelo de dupla-porosidade (Weber et al., 2001).

Figura 2. 15 - Transferência das propriedades equivalentes de fluxo do sistema de fraturas e falhas para o modelo de dupla-porosidade do campo (Bourbiaux et al., 2005). 50

Figura 2. 16 - Efeito da tensão efetiva sobre o fechamento das fraturas Navarro (2012). .53

Figura 2. 17 - Mapa de distribuição de tensão de compressão: máxima (em vermelho) e mínima (em verde) (Weber et al., 2001). .53

Figura 2. 18 - Geração de fraturas nas zonas de compressão mínima da Figura 2.16 (Weber et al., 2001). .54

Figura 2. 19 - Exemplo da discretização dos dois meios matriz e fraturas dentro de cada célula do grid (grid block) (Adaptado de Gilman e Kazemi, 1988). 55

Figura 2.20 - Representação do meio fissurado (Barenblatt et al.,1960). .57

Figura 2.21 - Idealização das heterogeneidades do meio poroso (Warren e Root, 1963). 58

Figura 2.22 - Representação do reservatório fraturado no modelo de duplaporosidade. Destaque para a sobreposição dos grids de fratura e de matriz (Lemonnier e Bourbiaux, 2010). 60

Figura 2. 23 - Detalhe do bloco de matriz cercado por fraturas (Paiva, 2012)...62 Figura 2. 24 - Fluxo viscoso por Gilman e Kazemi (1988) - Adaptado de Paiva (2012). 64

Figura 2. 25 - Representação do centro e das faces do bloco de matriz (Adaptado de Lemonnier e Bourbiaux, 2010). 66

Figura 2. 26 - Discretização vertical da célula da matriz em quatro subdomínios (IMEX - CMG, 2010). 69

Figura 2. 27 - Discretização do bloco de matriz e o esquema de comunicação de fluxo: a) modelo MINC; b) modelo de dupla-porosidade (Adaptado de Wu e Pruess, 1988). .70

Figura 2. 28 - Representação da célula de matriz utilizada por Naimi-Tajdar et al. (2007) com subdivisão tanto vertical quanto horizontal. 70

Figura 2.29 - Fluxograma de seleção do modelo de simulação para reservatórios naturalmente fraturados segundo a metodologia IFP (Bourbiaux, 2010)...72

Figura 3. 1 - Janela de construção do modelo de simulação do programa IMEX ${ }^{\circledR}$ (IMEX, 2010). 
Figura 3. 2 - Modelo Base para um reservatório naturalmente fraturado. $\quad 78$

Figura 3. 3 - Permeabilidades relativas do óleo e da água para a matriz. $\quad 81$

Figura 3. 4 - Permeabilidades relativas do gás e do óleo para a matriz. $\quad 81$

Figura 3. 5 - Curvas de pressão capilar para os sistemas óleo/água e óleo/gás para a matriz.

Figura 3. 6 - Hipótese de relação linear das permeabilidades relativas para 0 sistema de fraturas.

82

Figura 3. 7 - Estrutura do Modelo Corredor 1.

86

Figura 3.8 - (a) Seção de um grid de fraturas contendo corredores de fraturas e camadas de super-K; (b) Construção do modelo com corredor de fraturas (Uba et al., 2007).

Figura 3. 9 - Modelo Corredor 2 baseado na Figura 3. 8 (desconsiderando as camadas de super-K).

Figura 3. 10 - (a) Modelo apresentado por Uba et al. (2007) para análise da camada de super-K; (b) Modelo Corredor/Super-K; (c) Detalhe do grid de fraturas e da localização dos poços.

89

Figura 3. 11 - Seção da malha de simulação de um reservatório carbonático gigante (Fung e Al-Shaalan, 2005). 90

Figura 3. 12 - Grid do Modelo Campo GTEP representando metade do grid da Figura 3. 11.

Figura 3. 13 - Modelo do reservatório do Oriente Médio: (a) Irregularidade na varredura da água injetada; (b) Grid de fraturas, detalhe para a curvatura do modelo (Adaptadas de Cosentino et al., 2002). 92

Figura 3. 14 - Esquemas de produção aplicados ao Modelo Campo GTEP. 93

Figura 4. 1 - Curva de produção do Modelo DP.

Figura 4. 2 - Curva de produção do Modelo DPDK. 95

Figura 4. 3-Sequência da produção dos modelos DP (esquerda) e DPDK (direita) 96

Figura 4. 4 - Comportamento da produção acumulada de água para os modelos do Caso 1.

Figura 4. 5 - Comportamento da produção acumulada de água para os modelos do Caso 2A.

Figura 4. 6 - Comportamento da produção acumulada de água para os modelos do Caso 2B.

Figura 4.7 - Produção acumulada de água para os modelos do Caso 2B. 100 
Figura 4. 8 - Sequência da produção do Modelo Corredor 1 (direita) e de um modelo idêntico sem o corredor de fraturas (esquerda). 102

Figura 4. 9 - Vista inferior do fim da simulação do Modelo Corredor 1 (direita) e do modelo sem corredor de fraturas (esquerda).

Figura 4. 10 - Sequência de produção do Modelo Corredor 2. Detalhe para a trajetória de deslocamento da água. $\quad 104$

Figura 4. 11 - Comportamento da produção do Modelo Corredor/SuperK (vista superior da camada 18). 105

Figura 4. 12 - Comportamento irregular de varredura do Modelo Corredor/SuperK nos 5 primeiros anos de produção. 106

Figura 4. 13 - Comportamento da produção do modelo Corredor/Super-K. 107

Figura 4. 14 - Corte de água apresentado pelo Modelo Corredor/Super-K. 107

Figura 4. 15 - Comparação das respostas de pressão dos modelos de duplaporosidade DP, DPDK, SD e MINC. 108

Figura 4. 16 - Comparação do volume acumulado de água produzido pelos modelos DP, DPDK, SD e MINC. 109

Figura 4. 17 - Comparação do tempo de simulação dos modelos DP, DPDK, SD e MINC. 109

Figura 4. 18 - Comparação do Modelo Campo GTEP com o modelo de Cosentino et al. (2002).

Figura 4. 19 - Sequência de produção do Modelo Campo GTEP segundo dois esquemas diferentes de produção: esquema B (esquerda) e esquema C (direita). 


\section{Lista de tabelas}

Tabela 1 - Principais implicações de reservatórios naturalmente fraturados. 28

Tabela 2 - Propriedades dos meios adotadas no Modelo Base. 78

Tabela 3 - Propriedades PVT do fluido. $\quad 79$

Tabela 4 - Parâmetros adicionais dos fluidos. $\quad 80$

Tabela 5 - Casos gerados para a análise paramétrica. 83

Tabela 6 - Casos de continuidade capilar. 83

Tabela 7 - Caso de velocidade de transferência: permeabilidade da matriz. $\quad 84$

Tabela 8 - Caso de velocidade de transferência: espaçamento das fraturas. 84

Tabela 9 - Caso de condutividade hidráulica das fraturas. 84

Tabela 10 - Modificações do Modelo Base para o Modelo Corredor 1.

Tabela 11 - Parâmetros utilizados por Uba et al. (2007) para o reservatório Ghawar Arab-D. $\quad 88$

Tabela 12 - Parâmetros adotados para o Modelo Corredor 2.

Tabela 13 - Parâmetros adotados para o Modelo Corredor/Super-K. 89

Tabela 14 - Parâmetros adotados para o Modelo Campo GTEP. 92 


\section{Lista de nomenclaturas}

Letras latinas:

\begin{tabular}{|c|c|}
\hline$A$ & Área \\
\hline B & Fator volume de formação do fluido (ex. Bo, Bw, Bg) \\
\hline$c_{\text {matriz }}$ & Compressibilidade da estrutura rochosa \\
\hline$c_{b}$ & Compressibilidade volumétrica (bulk compressibility) \\
\hline E & Módulo de Young (ou de Elasticidade) \\
\hline Eg & Fator de expansão do gás (inverso do Bg) \\
\hline$F^{c}$ & Fator de ajuste do efeito capilar \\
\hline$F^{g}$ & Fator de ajuste do efeito da drenagem gravitacional \\
\hline$F^{v}$ & Fator de ajuste do efeito da drenagem viscosa \\
\hline$l_{a w}$ & Índice de Amott de molhabilidade à água (1 molhável; 0: não molhável) \\
\hline$l_{\text {cel }}$ & Escala da célula do grid (ex. $\Delta x$ ) \\
\hline$l_{f}$ & Escala da fratura \\
\hline$l x, l y, l z$ & Dimensões equivalentes do bloco de matriz \\
\hline$k$ & Permeabilidade \\
\hline$K$ & Permeabilidade absoluta \\
\hline$\overline{K_{f}}$ & Permeabilidade equivalente da fratura \\
\hline$k_{r}$ & Permeabilidade relativa do fluido \\
\hline$m$ & Velocidade do fluido \\
\hline $\mathrm{n}$ & Número de blocos de matriz dentro de uma célula do grid \\
\hline $\mathrm{N}$ & Número de dimensões de fluxo (uni, bi ou tridimensional) \\
\hline$P$ & Pressão de poros \\
\hline $\mathrm{P}_{\mathrm{c}}$ & Pressão capilar \\
\hline$R_{S}$ & Razão de solubilidade do gás no óleo \\
\hline q & Vazão de fluidos \\
\hline S & Saturação do fluido \\
\hline $\mathrm{t}$ & Tempo \\
\hline te & Tempo de equilíbrio estático para o fluxo entre matriz e fratura \\
\hline V & Volume \\
\hline $\bar{w}$ & Ponderador médio das propriedades de interface das células \\
\hline Z & Profundidade de análise \\
\hline
\end{tabular}


Letras gregas:

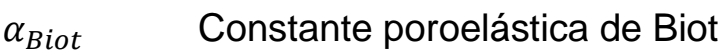

$\gamma \quad$ Peso específico do fluido

$\Delta p \quad$ Variação de pressão (pressão atual menos a pressão original)

$\Delta t \quad$ Tempo do passo da simulação

$\Delta x, \Delta y, \Delta z \quad$ Dimensões de uma célula de simulação

$\lambda \quad$ Mobilidade do fluido

$\mu \quad$ Viscosidade dinâmica do fluido

$\rho \quad$ Densidade

$\sigma \quad$ Fator de forma (referente às dimensões do bloco de matriz)

$\sigma^{\prime} \quad$ Tensão efetiva

$\sigma_{T} \quad$ Tensão total

$\tau \quad$ Termo de transferência de fluxo entre matriz e fraturas

$v \quad$ Coeficiente de Poisson

$\phi \quad$ Porosidade

$\Phi \quad$ Potencial de fluxo (potencial de pressão + potencial de elevação)

Sobrescrito:

$\wedge$

Representa os termos da fratura (ex. porosidade da fratura, $\hat{\phi}$ )

H Horizontal

V Vertical

\section{Subscrito:}

$\alpha \quad$ Fase (óleo, água ou gás)

$\xi \quad$ Componente do fluido

C $\quad$ Centro do bloco de matriz

f Fratura

m Matriz

o Óleo

g Gás

S $\quad$ Face do bloco de matriz

sd Subdomínio

w Água

1 Fratura

$2 \quad$ Matriz 
Siglas:

$\mathrm{BHI} \quad$ Borehole Images - perfis de imagem do poço

DFN Discrete Fracture Network (Rede Discreta de Fraturas)

DFM Discrete Fracture and Matriz ou Discrete Fracture Model

DP Dupla-porosidade

DPDK Dupla-porosidade e dupla-permeabilidade

GTEP Grupo de Tecnologia e Engenharia de Petróleo

MINC Multiple Interacting Continua - modelo de múltiplos domínios

OOIP Original oil in place - volume original de óleo presente na rocha

PVT Pressão, volume e temperatura

RB Reservoir Barrel - barril nas condições do reservatório

scf Standard cubic feet ( $\mathrm{ft}^{3}$ em condições de superfície)

SD Subdomínios

STB Stock Tank Barrel - barril em condições de superfície (standard) 